\title{
$\gamma$-Aminobutyric acid induces transcriptional changes contributing to salt tolerance in creeping bentgrass
}

\author{
Z. LI*, B.Z. CHENG, Y. PENG, and Y. ZHANG \\ Department of Grassland Science, Animal Science and Technology College, Sichuan Agricultural University, \\ Chengdu 611130, P.R. China
}

\begin{abstract}
$\gamma$-Aminobutyric acid (GABA) regulates plant tolerance to abiotic stresses; however, a transcriptomic change and key stressrelated genes induced by GABA have not been investigated in plants during a prolonged period of salt stress. Roots of creeping bentgrass (Agrostis stolonifera) cv. Penncross were pretreated with or without $0.5 \mathrm{mM}$ GABA solution for 2 days and then subjected to salt stress for 20 days $(150 \mathrm{mM} \mathrm{NaCl}$ solution for $3 \mathrm{~d}, 200 \mathrm{mM} \mathrm{NaCl}$ for another $3 \mathrm{~d}$, and $250 \mathrm{mM}$ $\mathrm{NaCl}$ for $14 \mathrm{~d}$ ) in controlled growth chambers. The application of GABA significantly increased GABA content in roots and alleviated a salt-stress induced decrease in GABA content in leaves. This was associated with a significant increase in salt tolerance as demonstrated by a significantly higher leaf relative water content, photochemical efficiency, performance index on absorption basis, and lower electrolyte leakage in GABA-pretreated plants as compared to untreated plants under salt stress. Transcriptomic analysis found that GABA-induced salt tolerance was closely associated with saccharide, amino acid, and lipid metabolism. The GABA upregulated key differentially expressed genes including cytochrome P450 (CYP450), zinc transporter 29 (ZTP29), alpha-amylase 3 (AMY3), 3-ketoacyl-CoA synthase 6 (KCS6), aldehyde oxidase $(A O)$, acetyl-CoA carboxylase 1 ( $A C C 1)$, and magnesium-chelatase $(\mathrm{Mg}-\mathrm{CHT})$ involved in zinc homeostasis, starch degradation, and the biosynthesis of wax, fatty acid, chlorophyll, and abscisic acid, which could contribute to GABAregulated salt tolerance. Current findings prove that GABA application is an efficient approach to enhance salt tolerance of creeping bentgrass during a prolonged period of salt stress and also provide valuable information to better understand key candidate genes and regulatory pathways of GABA-induced salt tolerance in plants.
\end{abstract}

Additional key words: abscisic acid, amino acid, fatty acid, lipid metabolism, starch degradation, wax, zinc homeostasis.

\section{Introduction}

Soil salinity has become a serious threat to agricultural sustainable development, since over $6 \%$ of total land area in the world is affected by salt stress (Flowers et al. 2010). Physiological, biochemical, and molecular changes in plants are negatively impacted by salt stress due to low osmotic potential in soil, ion toxicity, and secondary damage including nutritional imbalance and oxidative stress (Yang and Guo 2018). Salt tolerance like tolerance to other abiotic stress is regulated by multiple genes in plants. Transcriptomics provides an effective strategy to detect extensive gene expressions helping to understand complex mechanism of salt tolerance (Nagalakshmi et al. 2010). Many candidate genes associated with stress signal transduction, ion transport, osmolytes synthesis, antioxidant defense, and hormonal regulation contributing to salt tolerance have been found or identified in various plant species based on RNA-seq method or other biotechnological methods (Muchate et al. 2016). Recent study found out that differentially expressed genes (DEGs) related to $\mathrm{Ca}^{2+}$ signal transduction and abscisic acid (ABA) pathway took part in regulating salt tolerance when Camellia sinensis was subjected to $4 \mathrm{~h}$ of salt stress (Wan et al. 2018). Transcriptomic analysis also proved that DEGs involved in ion transport and antioxidant defense played vital roles in Betula halophila in response to $24 \mathrm{~h}$ of salt stress (Shao et al. 2018). Although a large number

\section{Submitted 17 July 2020, last revision 5 August 2020, accepted 10 August 2020.}

Abbreviations: ABA - abscisic acid; ABCB4 - ABC transporter B family member 4; ACC1 - acetyl-CoA carboxylase 1; AMY3 alpha-amylase 3; AO - aldehyde oxidase; CYP450 - cytochrome $\mathrm{P}_{450}$; CRK10 or 39 - cysteine-rich receptor-like protein kinase 10 or 39; CLASP - CLIP-associated protein; Chi1 - endochitinase 1; DEGs - differentially expressed genes; EL - electrolyte leakage; $F_{v} / F_{m}$ - photochemical efficiency; GABA - $\gamma$-aminobutyric acid; GO - gene ontology; KEGG - Kyoto encyclopedia of genes and genomes; KCS6 - 3-ketoacyl-CoA synthase 6; Mg-CHT - magnesium-chelatase; PIABS - performance index on absorption basis; RWC - relative water content; ZTP29 - zinc transporter 29.

Acknowledgments: This research was supported by the National Natural Science Foundation of China (31702182) and the China Postdoctoral Science Foundation (2018M630086). The first two authors contributed equally to the manuscript.

* Corresponding author; e-mail: lizhou1986814@163.com. 
of researches have focused on examining changes of gene transcriptions during a short-term salt stress (several hours or days), the transcriptional regulation has not been well documented when plants respond to a long-term salt stress (more than $20 \mathrm{~d}$ ).

With a global climate change, crops are suffering from more harsh environmental condition including salt stress. Creeping bentgrass (Agrostis stolonifera) is one of the most important and desirable turf grasses being used in golf course worldwide. As a glycophyte, it has lower salt tolerance than other turfgrass species such as bermudagrass (Marcum 2001, Tran et al. 2018). The accelerated soil salinization due to the extensive use of reclaimed water, fertilizers, and pesticides, especially in coastal areas, decreases turf quality (Duan et al. 2009). Some possible mechanisms of adaptation to salt stress have been uncovered in creeping bentgrass. For example, increased leaf wax content and reduced sodium uptake are crucial for enhanced salt tolerance in transgenic creeping bentgrass overexpressing miR319 (Zhou et al. 2013). The accumulation of plant growth regulators (PGRs) such as abscisic acid (ABA), jasmonic acid (JA), salicylic acid (SA), and polyamines (PAs) are beneficial for creeping bentgrass to survive salt stress (Krishnan and Merewitz 2015, Ma and Merewitz 2016). In response to salt stress, the relatively salt-tolerant creeping bentgrass cultivar Penn-A4 has higher protein abundance associated with antioxidant defense and amino acids metabolism compared with salt-sensitive cv. Penncross (Xu et al. 2010). Previous studies have used transcriptome to explore key genes and metabolic pathways in creeping bentgrass when exposed to heat and drought stress (Xu et al. 2017, Li et al. 2019). However, transcriptomic analysis has not been applied to unveil the mechanism of salt tolerance in creeping bentgrass.

$\gamma$-Aminobutyric acid (GABA) is known to be a nonprotein amino acid participating in plant growth regulation, nutrition, and adaptation to abiotic stresses (Tarkowski et al. 2020). It has been reported that the positive role of GABA in regulating tolerance to abiotic stress is associated with organic metabolites accumulation and lower lipid peroxidation in creeping bentgrass and perennial ryegrass under drought stress (Krishnan et al. 2013, Li et al. 2017b), less damage to photosynthetic machinery in mungbean plants under heat stress (Priya et al. 2019), and better photosynthetic performance and less oxidative damage in pepper seedlings under low irradiance (Li et al. 2017a). Recent studies also have found out that GABA played diverse roles in regulating salt tolerance in different plant species. Exogenous GABA mitigates adverse effects of salt stress on maize seedlings through improving antioxidant enzyme activities to alleviate oxidative damage (Wang et al. 2017). The GABA priming significantly enhances salt tolerance of white clover during seed germination mainly due to accelerated starch metabolism and improved antioxidant defense (Cheng et al. 2018). Salt stress promotes GABA accumulation and metabolism that provided an available carbon source for energy supply and defensive responses (Che-Othman et al. 2020). Water, ions, and metabolites homeostasis contribute to GABA- induced salt tolerance in creeping bentgrass based on proteomic and metabolomic analyses (Li et al. 2020). In spite of these previous studies, the GABA-induced change of transcriptome in response to a prolonged period of salt stress is not reported yet. Therefore, objectives of this study were to investigate effects of exogenous application of GABA on improving salt tolerance based on physiological analysis and to determine key candidate genes and metabolic pathways regulated by GABA under salt stress via the transcriptome analysis. Current findings could help to elucidate GABA-induced mechanism of transcriptional regulation underlying the adaptation to salt stress in perennial turfgrass.

\section{Materials and methods}

Plants and treatments: Seeds of creeping bentgrass (Agrostis stolonifera L. cv. Penncross) were purchased from Tee-2-Green Corporation (Hubbard, OR, USA) and germinated in containers $(30 \mathrm{~cm}$ length, $15 \mathrm{~cm}$ width, and $20 \mathrm{~cm}$ height) filled with quartz sand. After being germinated in distilled water for $10 \mathrm{~d}$, seedlings were cultivated in Hoagland's nutrient solution (Hoagland and Arnon 1950) for $20 \mathrm{~d}$. The growth chambers (GXZ500C, Ningbo Jiangnan Instrument Factory, Ningbo, China) were set to day/night temperatures of $23 / 19^{\circ} \mathrm{C}$, a $70 \%$ relative humidity, and a $700 \mu \mathrm{mol} \mathrm{m} \mathrm{m}^{-2} \mathrm{~s}^{-1}$ photosynthetically active radiation. For the GABA ( $\geq 99 \%$, Sigma-Aldrich, St. Louis, USA) pretreatment, plants were cultivated in the Hoagland's nutrient solution containing $0.5 \mathrm{mM}$ GABA for $2 \mathrm{~d}$ and non-GABA-treated plants were cultivated in standard Hoagland's nutrient solution for $2 \mathrm{~d}$. The GABA-pretreated or untreated plants were then subjected to $150 \mathrm{mM} \mathrm{NaCl}$ solution for $3 \mathrm{~d}$, $200 \mathrm{mM} \mathrm{NaCl}$ for another $3 \mathrm{~d}$, and $250 \mathrm{mM} \mathrm{NaCl}$ for $14 \mathrm{~d}$ as the treatments SSG or SS, respectively. The control C without salt stress and GABA pretreatment was cultivated in Hoagland's nutrient solution for $22 \mathrm{~d}$. Each treatment had four replications (four containers). Hoagland solutions were refreshed every day and aerated by using aeration pumps $\left(115 \mathrm{~V}, 60 \mathrm{~Hz}\right.$, Tetra ${ }^{\circledR}$ Blacksburg, Blacksburg, VA, USA). Four or three independent replications were used for analyzing physiological parameters or transcriptome, respectively. Leaves were sampled at 0,10 , and $20 \mathrm{~d}$ during salt stress.

Measurement of physiological parameters: For detecting leaf relative water content (RWC), fresh leaves $(0.2 \mathrm{~g})$ were collected from plants and fresh mass (FM) was determined. These leaves were then immerged in deionized water for $12 \mathrm{~h}$ and water saturated mass (WSM) was obtained. Then, the leaves were dried in an oven at $80{ }^{\circ} \mathrm{C}$ for $72 \mathrm{~h}$ to a constant mass (DM). RWC [\%] = [(FM - DM)/(WSM - DM)] × 100 (Barrs and Weatherley 1962). For the determination of electrolyte leakage (EL), $0.15 \mathrm{~g}$ of fresh leaves was cleaned with deionized water for three times and then immerged in $45 \mathrm{~cm}^{3}$ of deionized water for $24 \mathrm{~h}$. The initial conductance of leaching solution was determined by using a conductivity meter ( $D D S$ - 
307A, INESA Scientific Instrument Company, Shanghai, China). The maximum conductance of the leaching solution was determined after leaves were autoclaved at $120{ }^{\circ} \mathrm{C}$ for $15 \mathrm{~min}$ and cooled to room temperature (Blum and Ebercon 1981) and EL was calculated. For the determination of photochemical efficiency (determined as variable to maximum chlorophyll fluorescence, $\mathrm{F}_{\mathrm{v}} / \mathrm{F}_{\mathrm{m}}$ ) and performance index on absorption basis (PIABS), a single leaf was adapted to darkness for 30 min by using leaf clips, and the $\mathrm{F}_{\mathrm{v}} / \mathrm{F}_{\mathrm{m}}$ and PIABS were recorded with the fluorescence meter (Pocket PEA, Hansatech, Norfolk, UK). The PIABS is a photosynthetic parameter that reflects the photosynthetic performance and health status of leaves. The GABA content was detected by using the assay kits purchased from the Shanghai Enzyme-linked Biotechnology Co., Shanghai, China.

Transcriptome analysis: The $0.1 \mathrm{~g}$ of fresh leaves were collected and immediately immersed in liquid nitrogen. For total RNA extraction, an RNeasy Mini kit (Qiagen, Düsseldorf, Germany) was used according to the manufacturer's instructions. For the cDNA library construction, the mRNA-Seq sample preparation kit ${ }^{\mathrm{TM}}$ (Illumina, San Diego, CA, USA) was used and specific methods have been described in details in our previous studies (Li et al. 2017c). Transcriptome assembly was carried out with short reads assembling program (Trinity, $v r 20131110$ ), and then the distribution of length of contigs and unigenes were calculated (Haas et al. 2013). The gene relative expression ratio was obtained according to different comparison groups ( $\mathrm{SS}$ vs. C, SSG vs. C, and SSG vs. SS), and the $\log _{2}$ fold change of $>1$ or $<$ -1 were considered as DEGs. The DEGs were classified and annotated with the databases of nonredundant NCBI protein sequences (NR, https://www.ncbi.nlm.nih.gov/), nonredundant NCBI nucleotide sequences (NT, https:// www.ncbi.nlm.nih.gov/), a manually annotated and reviewed protein sequence database (Swiss-Prot, http:// www.ebi.ac.uk/uniprot/), gene ontology (GO, http://www. geneontology.org/), and Kyoto encyclopedia of gene and genomes (KEGG, http://www.genome.jp/kegg/).
Statistical analysis: The significance of effects of salt stress and salt stress plus GABA on physiological parameters was analyzed by using the general linear model procedure of $S A S$ (v. 9.1; SAS Institute, Cary, NC). Significant differences were tested by using analysis of variance $(A N O V A)$ followed by the least significance difference test (LSD) with $\alpha=0.05$ at a given day.

\section{Results}

Phenotypic changes of creeping bentgrass in response to GABA pretreatment and after $20 \mathrm{~d}$ of salt stress are shown on Fig. $1 A$. The GABA content in roots significantly increased in the SS and SSG compared with that in the C (Fig. 1B). The SSG treatment exhibited $22 \%$ increase in GABA content in roots than the SS treatment. Salt stress significantly decreased GABA content in leaves, whereas the GABA content in leaves of salt-stressed plants pretreated with GABA maintained on control level on $20^{\text {th }}$ day of salt stress (Fig. $1 B$ ). Leaf RWC, EL, $\mathrm{F}_{\mathrm{v}} / \mathrm{F}_{\mathrm{m}}$, and PIABS did not change by the GABA pretreatment at 0 day (Fig. 2). The RWC, $F_{v} / F_{m}$, and PIABS significantly decreased and EL increased gradually in both of GABAtreated and untreated plants during salt stress. The SSG treatment exhibited 16 and $24 \%$ increases in RWC than the SS treatment at 10 and $20 \mathrm{~d}$, respectively (Fig. 2A). The GABA-pretreated plants had 24 or $25 \%$ decrease in EL as compared to untreated plants at 10 or $20 \mathrm{~d}$ of salt stress, respectively (Fig. $2 B$ ). Although salt stress induced significant declines in $\mathrm{F}_{\mathrm{v}} / \mathrm{F}_{\mathrm{m}}$ in both of GABA-pretreated and untreated plants, the plants under SSG treatment maintained significantly higher $\mathrm{F}_{\mathrm{v}} / \mathrm{F}_{\mathrm{m}}$ than under the SS treatment at 10 and $20 \mathrm{~d}$ (Fig. 2C). A 33 or $67 \%$ increase in PIABS was observed in the SSG as compared to that in the SS at 10 or $20 \mathrm{~d}$, respectively (Fig. $2 D$ ).

Fig. $3 A$ showed the heat map of DEGs in SS vs. C, SSG vs. C, and SSG vs. SS. The GABA application altered the DEGs profiling as reflected by the difference in DEGs between SS vs. C and SSG vs. C (Fig. 3A). A total of 14857,14649 , or 245 DEGs was identified in SS

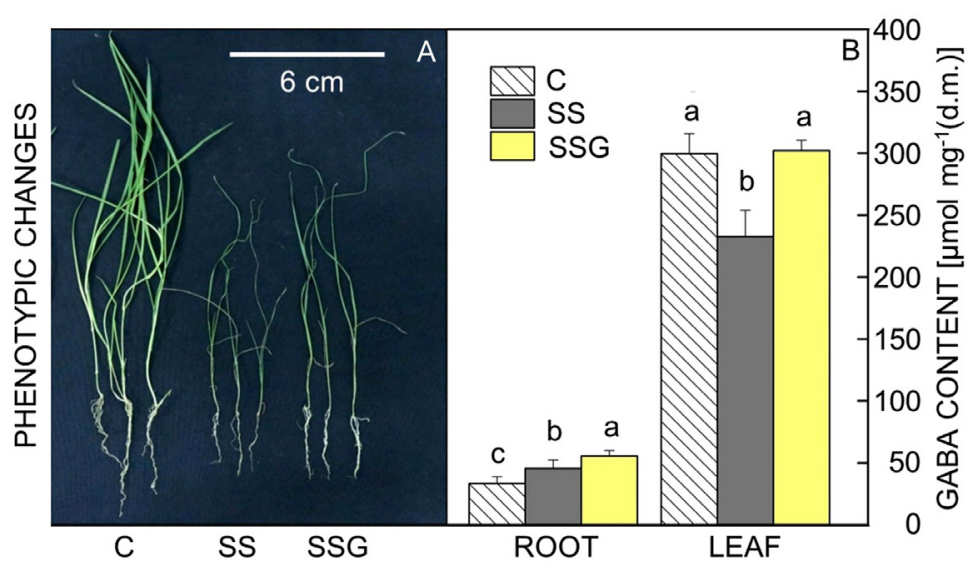

Fig. 1. Effects of salt stress and exogenous $\gamma$-aminobutyric acid (GABA) on a phenotypic change $(A)$ and GABA content $(B)$ in creeping bentgrass at $20 \mathrm{~d}$ of salt stress. C - control, SS - salt stress, SSG - salt stress plus $0.5 \mathrm{mM} \mathrm{GABA}$. Means \pm SEs, $n=4$; different letters indicate significant differences at $P<0.05$. 


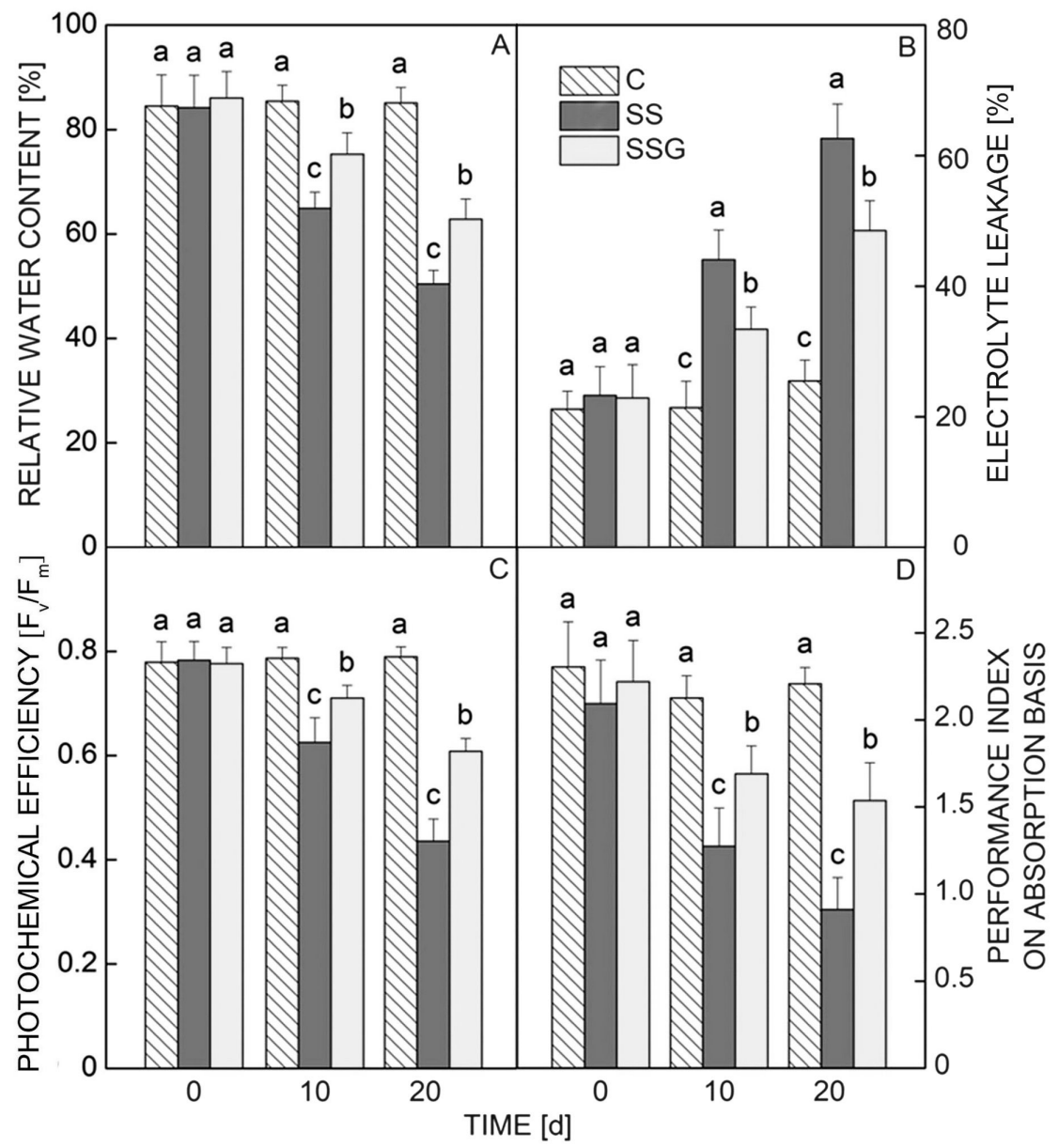

Fig. 2. Effects of salt stress and exogenous $\gamma$-aminobutyric acid (GABA) on relative water content $(A)$, electrolyte leakage $(B)$, photochemical efficiency $(C)$, and performance index on absorption basis (PIABS, $D$ ) in creeping bentgrass. C - control, SS - salt stress, SSG - salt stress plus $0.5 \mathrm{mM}$ GABA. Means \pm SEs, $n=4$; different letters indicate significant differences at $P<0.05$.

vs. C, SSG vs. C, and SSG vs. SS, respectively (Fig. 3B). A total of 120 DEGs was commonly detected in SS vs. C, SSG vs. C, and SSG vs. SS; The SS vs. C and SSG vs. $\mathrm{C}$ had 1191 common DEGs; a total of 3 494, 3 301, or 36 DEGs specially identified in SS vs. C, SSG vs. C, or SSG vs. SS, respectively (Fig. 3C). Based on $G O$ analysis, most of DEGs were involved in protein metabolic process, phosphorus metabolic process, phosphate-containing compound metabolic process, and macromolecule modification, located in transcription factor complex and photosystem I, and had transferase activity, ion binding, phosphotransferase activity, kinase activity, and protein kinase activity (Fig. 1 Suppl.). The pathway enrichment analysis of DEGs found that most of DEGs enriched in in SS vs. C and SSG vs. C including phenylpropanoid biosynthesis, plant hormone signal transduction, starch and sucrose metabolism, photosynthesis, and galactose metabolism (Fig. 4). The glycerolipid metabolism and diterpenoid biosynthesis were only found in SSG vs. C (Fig. 4B). The KEGG analysis of DEPs found that most of DEGs were involved in saccharide metabolism, lipid metabolism, translation, biosynthesis of other secondary metabolites, amino acid metabolism, and environmental adaptation in SSG vs. SS (Fig. 5).

Fig. 6 showed 12 up-regulated DEGs associated with salt tolerance or plant growth and development in $\mathrm{SSG}$ vs. SS including cytochrome P450 (CYP450), 3-ketoacylCoA synthase 6 (KCS6), endochitinase 1 (Chil), aldehyde oxidase (AO), zinc transporter 29 (ZTP29), cysteine-rich receptor-like protein kinase 10 (CRK10), CRK39, acetyl$C o A$ carboxylase 1 (ACC1), ABC transporter B family member 4 (ABCB4), magnesium-chelatase (Mg-CHT), alpha-amylase 3 (AMY3), and CLIP-associated protein (CLASP).

\section{Discussion}

High salt-induced osmotic stress leads to decrease in RWC and stomatal closure, which causes a significant decline in photosynthesis (Muchate et al. 2016). Physiological analyses found that exogenous GABA significantly alleviated salt-induced declines in endogenous GABA content, RWC, $\mathrm{F}_{\mathrm{v}} / \mathrm{F}_{\mathrm{m}}$, and PIABS, and also improved cell membrane stability in leaves during a prolonged period of salt stress. These findings are consistent with a previous 

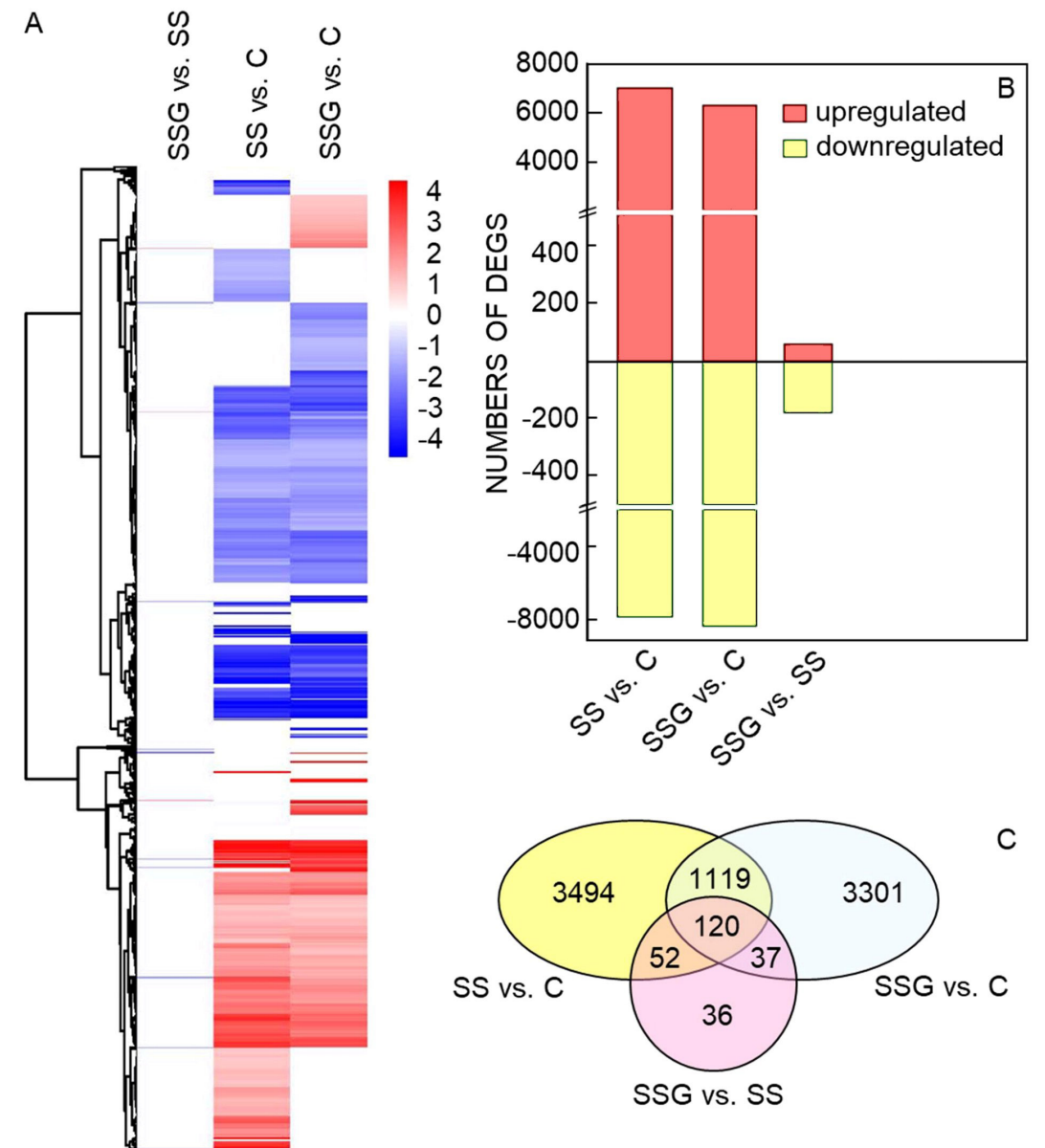

Fig. 3. The heat map of differentially expressed genes (DEGs, $A$ ), total numbers of DEGs $(B)$, and Venn diagram comparing the number of DEGs $(C)$ in leaves of creeping bentgrass in response to salt stress and $\gamma$-aminobutyric acid. C - control, SS - salt stress, SSG - salt stress plus $0.5 \mathrm{mM}$ GABA.

study about beneficial effects of GABA on improving salt tolerance of maize seedlings (Wang et al. 2017). The transcriptomic results showed that salt stress induced significant changes of some common metabolic pathways in GABA-pretreated and untreated creeping bentgrass including phenylpropanoid biosynthesis, plant hormone signal transduction, starch and sucrose metabolism, and photosynthesis. The activation or up-regulation of these pathways has been reported to be closely related to improved salt tolerance in plants (Krishnan and Merewitz 2015, Muchate et al. 2016, Rossi et al. 2016). In our previous study we also found that phenylpropanoid biosynthesis, plant hormone signal transduction, and carbon metabolism were main metabolic processes involved in the adaptation to heat stress and GABA-regulated heat tolerance in creeping bentgrass (Li et al. 2019). Interestingly, the DEGs concerning glycerolipid metabolism was only found in SSG vs. C. In addition, most of DEGs in SSG vs. SS were involved in saccharide, amino acid, and lipid metabolism. The accumulation of sugars and amino acids induced by GABA contributing to enhanced salt tolerance has been reported in creeping bentgrass (Li et al. 2020). Dong et al. (2019) show that phenylpropanoid biosynthesis and glycerolipid metabolism play crucial roles in endophyte- induced salt tolerance in Arabidopsis. Choline priming significantly improves salt tolerance of Paspalum vaginatum via the up-regulation of lipid metabolism (Gao et al. 2019). Current findings indicate that GABA-induced salt tolerance is closely associated with saccharide, amino acid, and lipid metabolism in creeping bentgrass. These findings further reveal a positive role of GABA in regulating lipid metabolism in relation to salt tolerance in plants, which has not been investigated in previous studies.

Transcriptions of genes depend on stress severity and duration. Some key DEGs including CYP450, ZTP29, $A M Y 3, K C S 6, A O, A C C 1$, and $M g-C H T$ were significantly up-regulated by application of GABA in creeping bentgrass at $20 \mathrm{~d}$ of salt stress. It has been found out that a CYP450 mutant of Arabidopsis is sensitive to salt stress owing to metabolic disturbance, but the transgenic CYP450 mutant with a wild type CYP450 complemented a loss of salt tolerance (Mao et al. 2013). ZTP29 plays a vital role in enhancing salt tolerance through regulating zinc homeostasis in Arabidopsis (Wang et al. 2011), and ZTP29 Arabidopsis mutant showed hypersensitivity to salt stress, which further indicates the positive function of ZTP29 in salt tolerance (Wang et al. 2010). AMY3 is important for stress-induced starch degradation and tolerance to 


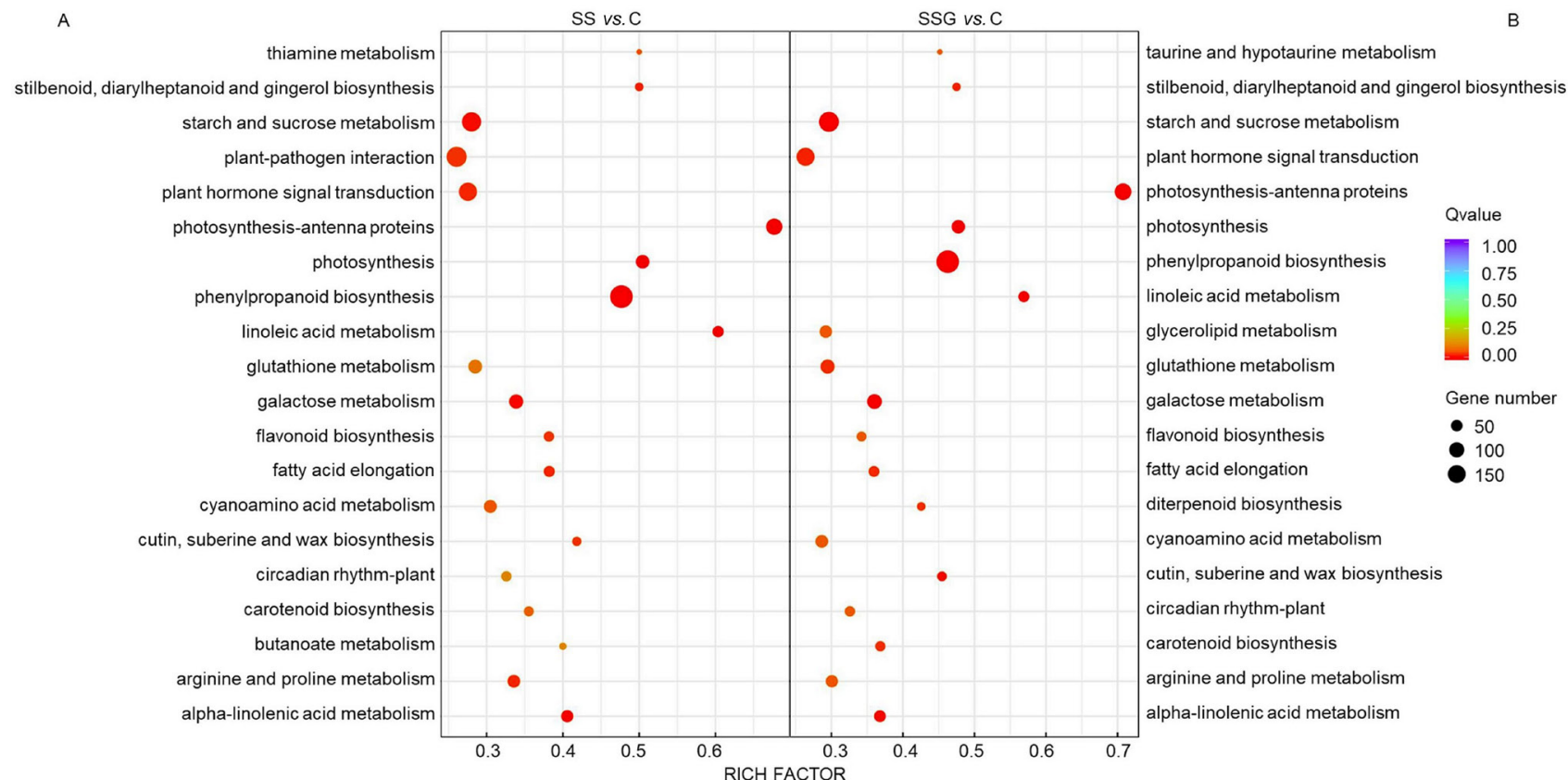

Fig. 4. The pathway enrichment analysis of identified differentially expressed genes in SS vs. C $(A)$ and SSG vs. C (B). C - control, SS - salt stress, SSG - salt stress plus $0.5 \mathrm{mM} \gamma$-aminobutyric acid.

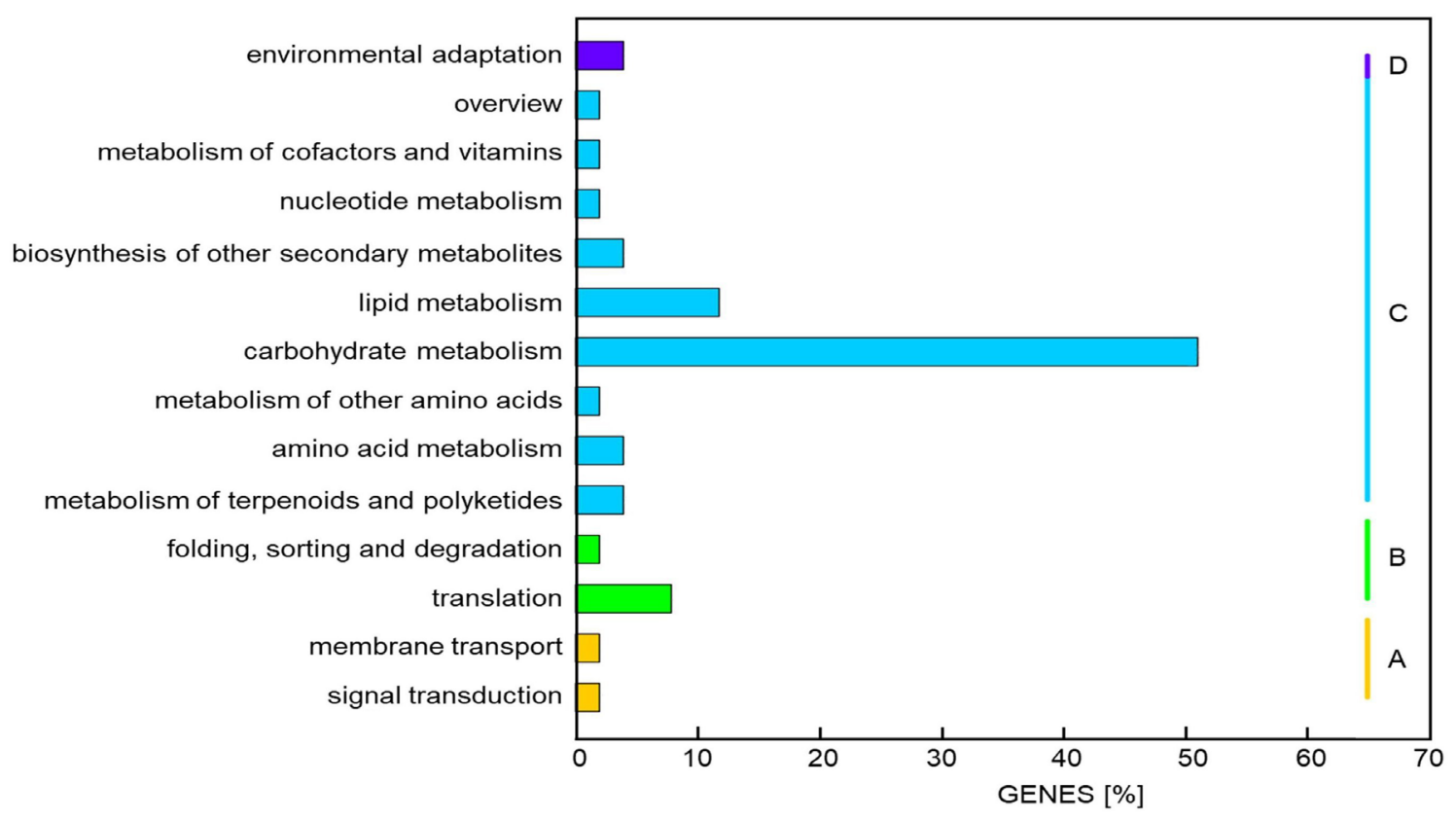

Fig. 5. The Kyoto encyclopedia of genes and genomes analysis of identified differentially expressed genes in salt stress plus $0.5 \mathrm{mM}$ $\gamma$-aminobutyric acid $v s$. salt stress. A - environmental information processing, B - genetic information processing, C metabolism, D systems of organisms.

osmotic stress in plants (Thalmann et al. 2016). It has been found out that GABA priming could significantly improve AMY activity leading to the alleviation of salt-induced inhibition of starch degradation during white clover seeds germination (Cheng et al. 2018). The KCS is involved in wax biosynthesis and increased wax content in leaf is positively related to salt tolerance in creeping bentgrass (Todd et al. 1999, Zhou et al. 2013). The improvement of AO activity or $A O$ gene expression provided protection against salt stress because AO catalyzed ABA synthesis inducing stomatal closure and so improving water balance in plants (Omarov et al. 1998, Szepesi et al. 2009, Horváth et al. 2011). ACC catalyzes the first step of fatty acids synthesis and mediates oxidative damage in plants (Zhang et al. 2012). Mg-CHT is a vital enzyme catalyzing chlorophyll biosynthesis and the maintenance of $\mathrm{Mg}$ - $\mathrm{CHT}$ expression 


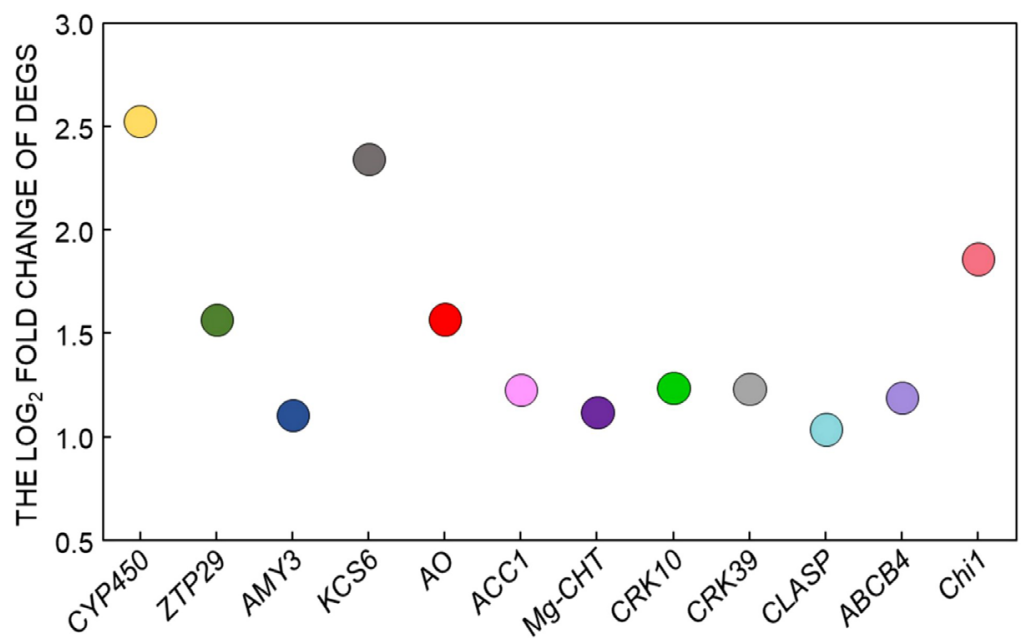

Fig. 6. Up-regulated differentially expressed genes (DEGs) in salt stress plus $0.5 \mathrm{mM} \gamma$-aminobutyric acid vs. salt stress. CYP450 cytochrome P450, ZTP29 - zinc transporter 29, AMY3 - alpha-amylase 3, KCS6 - 3-ketoacyl-CoA synthase 6, AO - aldehyde oxidase, ACC1 - acetyl-CoA carboxylase 1, Mg-CHT - magnesium-chelatase, CRK10 or 39 - cysteine-rich receptor-like protein kinase 10 or 39 , CLASP - CLIP-associated protein, ABCB4 - ABC transporter B family member 4, Chi1 - endochitinase 1.

is conducive to the alleviation of leaf senescence in white clover under osmotic stress (Zhao et al. 2020). Our current findings together with previous evidences indicated that zinc homeostasis, starch degradation, and the biosynthesis of wax, fatty acid, chlorophyll, and ABA could contribute to GABA-regulated salt tolerance in creeping bentgrass.

Significantly up-regulated DEGs CRK10, CRK39, CLASP, $A B C B 4$, and Chil were observed in SSG vs. SS in the current study. $C R K S$ are essential regulators of plant development and adaptation to stressful environments in Arabidopsis and the overexpression of CRK5 in Arabidopsis confers drought tolerance through regulating ABA signaling (Lu et al. 2016). CLASP regulates cells division, the stabilization of dynamic microtubules, and subsequent cell shape in plants (Ambrose et al. 2007). The transport of auxin and roots development were mediated by $A B C B 4$ in Arabidopsis (Terasaka et al. 2005, Santelia et al. 2006). Chi catalyzes the degradation of chitosan into chito-oligosaccharide that widely takes part in plant growth and nutrition regulation (Poshina et al. 2018). However, the function of these genes in salt tolerance is still largely unknown. The CRK10, CRK39, CLASP, ABCB4, and Chil could be possible candidate genes related to GABAinduced salt tolerance in creeping bentgrass, but this needs to be verified in future study.

\section{Conclusions}

The application of GABA significantly increased endogenous GABA accumulation in roots and also complemented a loss of GABA in leaves which was associated with a significant increase in salt tolerance in creeping bentgrass. Transcriptomic analysis found out that GABA-induced salt tolerance was closely associated with saccharide, amino acid, and lipid metabolism. GABA-upregulated key DEGs including CYP450, ZTP29, $A M Y 3, K C S 6, A O, A C C 1$, and $M g-C H T$ involved in zinc homeostasis, starch degradation, and the biosynthesis of wax, fatty acid, chlorophyll, and ABA, respectively, could contribute to GABA-regulated salt tolerance. Current findings prove that GABA application is an efficient approach to enhance salt tolerance of creeping bentgrass during a prolonged period of salt stress and also provide valuable information to better understand key candidate genes and regulatory pathways of GABA-induced salt tolerance in plants.

\section{References}

Ambrose, J.C., Shoji, T., Kotzer, A.M., Pighin, J.A., Wasteneys, G.O.: The Arabidopsis CLASP gene encodes a microtubuleassociated protein involved in cell expansion and division. Plant Cell 19: 2763-2775, 2007.

Barrs, H.D., Weatherley, P.E.: A re-examination of the relative turgidity technique for estimating water deficits in leaves. Aust. J. biol. Sci. 15: 413-428, 1962.

Blum, A., Ebercon, A.: Cell membrane stability as a measure of drought and heat tolerance in wheat. - Crop Sci. 21: 43-47, 1981.

Che-Othman, M.H., Jacoby, R.P., Millar, A.H., Taylor, N.L.: Wheat mitochondrial respiration shifts from the tricarboxylic acid cycle to the GABA shunt under salt stress. - New Phytol. 225: 1166-1180, 2020.

Cheng, B., Li, Z., Liang, L., Cao, Y., Zeng, W., Zhang, X., Ma, X., Huang, L., Nie, G., Liu, W., Peng, Y.: The $\gamma$-aminobutyric acid (GABA) alleviates salt stress damage during seeds germination of white clover associated with $\mathrm{Na}^{+} / \mathrm{K}^{+}$transportation, dehydrins accumulation, and stress-related genes expression in white clover. - Int. J. mol. Sci. 19: 2520,2018.

Dong, Z.Y., Rao, M.P.N., Wang, H.F., Fang, B.Z., Liu, Y.H., Li, L., Xiao, M., Li, W.J.: Transcriptomic analysis of two endophytes involved in enhancing salt stress ability of Arabidopsis thaliana. - Sci. total Environ. 686: 107-117, 2019.

Duan, C.R., Carrow, N.R., Huck, T.M.: Turfgrass and Landscape Irrigation Water Quality: Assessment and Management. CRC Press, Boca Raton 2009.

Flowers, T.J., Galal, H.K., Bromham, L.: Evolution of halophytes: 
multiple origins of salt tolerance in land plants. - Funct. Plant Biol. 37: 604-612, 2010.

Gao, Y., Li, M., Zhang, X., Yang, Q., Huang, B.: Upregulation of lipid metabolism and glycine betaine synthesis are associated with choline-induced salt tolerance in halophytic seashore paspalum. - Plant Cell Environ. 43: 159-173, 2019.

Haas, B.J., Papanicolaou, A., Yassour, M., Grabherr, M., Regev, A.: De novo transcript sequence reconstruction from RNASeq using the Trinity platform for reference generation and analysis. - Nat. Protocols 8: 1494-1512, 2013.

Hoagland, D.R., Arnon, D.I.: The water-culture method for growing plants without soil. - Calif. Agr. Exp. Sta. Circular 347: 357-359, 1950.

Horváth, E., Gallé, G., Szepesi, G., Tari, I., Csiszár, J.: Changes in aldehyde oxidase activity and gene expression in Solanum lycopersicum L. shoots under salicylic acid pre-treatment and subsequent salt stress. - Acta biol. szegediensis 55: 83-85, 2011.

Krishnan, S., Laskowski, K., Shukla, V., Merewitz, E.B.: Mitigation of drought stress damage by exogenous application of a non-protein amino acid $\gamma$-aminobutyric acid on perennial ryegrass. - J. amer. Soc. hort. Sci. 138: 358-366, 2013.

Krishnan, S., Merewitz, E.B.: Phytohormone responses and cell viability during salinity stress in two creeping bentgrass cultivars differing in salt tolerance. - J. amer. Soc. hort. Sci. 140: 346-355, 2015.

Li, Y., Fan, Y., Ma, Y., Zhang, Z., Yue, H., Wang, L., Li, J., Jiao, Y.: Effects of exogenous $\gamma$-aminobutyric acid (GABA) on photosynthesis and antioxidant system in pepper (Capsicum annuum L.) seedlings under low light stress. - J. Plant Growth Regul. 36: 436-449, $2017 \mathrm{a}$.

Li, Z., Cheng, B., Zeng, W., Liu, Z., Peng, Y.: The transcriptional and post-transcriptional regulation in perennial creeping bentgrass in response to $\gamma$-aminobutyric acid (GABA) and heat stress. - Environ. exp. Bot. 162: 515-524, 2019.

Li, Z., Cheng, B., Zeng, W., Zhang, X., Peng, Y.: Proteomic and metabolomic profilings reveal crucial function of $\gamma$-aminobutyric acid (GABA) on regulating ionic, water, and metabolic homeostasis in creeping bentgrass under salt stress. - J. Proteome Res. 19: 769-780, 2020.

Li, Z., Yu, J., Peng, Y., Huang, B.: Metabolic pathways regulated by abscisic acid, salicylic acid and $\gamma$-aminobutyric acid in, association with improved drought tolerance in creeping bentgrass (Agrostis stolonifera). - Physiol. Plant. 159: 42-58, $2017 \mathrm{~b}$.

Li, Z., Zhang, Y., Zhang, X., Merewitz, E., Peng, Y., Ma, X., Huang, L., Yan, Y.: Metabolic pathways regulated by chitosan contributing to drought resistance in white clover. - J. Proteome Res. 16: 3039-3052, 2017c.

Lu, K., Liang, S., Wu, Z., Bi, C., Yu, Y.T., Wang, X.F., Zhang, D.P.: Overexpression of an Arabidopsis cysteine-rich receptor-like protein kinase, $C R K 5$, enhances abscisic acid sensitivity and confers drought tolerance. - J. exp. Bot. 67: 5009-5027, 2016.

Ma, Y., Merewitz, E.: Polyamine content changes in creeping bentgrass exposed to salt stress. - J. amer. Soc. hort. Sci. 141: 498-506, 2016.

Mao, G., Seebeck, T., Schrenker, D., Yu O.: CYP709B3, a cytochrome P450 monooxygenase gene involved in salt tolerance in Arabidopsis thaliana. - BMC Plant Biol. 13: 169, 2013.

Marcum, K.B.: Salinity tolerance of 35 bentgrass cultivars. HortScience 36: 374-376, 2001.

Muchate, N.S., Nikalje, G.C., Rajurkar, N.S., Suprasanna, P., Nikam, T.D.: Plant salt stress: adaptive responses, tolerance mechanism and bioengineering for salt tolerance. - Bot. Rev. 82: 371-406, 2016.
Nagalakshmi, U., Waern, K., Snyder, M.: RNA-Seq: A method for comprehensive transcriptome analysis. - Curr. Protocols mol. Biol. 89: 1-13, 2010.

Omarov, R.T., Sagi, M., Lips, H.: Regulation of aldehyde oxidase and nitrate reductase in roots of barley (Hordeum vulgare L.) by nitrogen source and salinity. - J. exp. Bot. 49: 897-902, 1998.

Poshina, D.N., Raik, S.V., Poshin, A.N., Skorik, Y.A.: Accessibility of chitin and chitosan in enzymatic hydrolysis: a review. - Polymer Degrad. Stabil. 156: 269-278, 2018.

Priya, M., Sharma, L., Kaur, R., Bindumadhava, H., Nayyar, H.: GABA ( $\gamma$-aminobutyric acid), as a thermo-protectant, to improve the reproductive function of heat-stressed mungbean plants. - Sci. Rep. 9: 7788, 2019.

Rossi, L., Borghi, M., Francini, A., Lin, X., Xie, D.Y., Sebastiani, L.: Salt stress induces differential regulation of the phenylpropanoid pathway in Olea europaea cultivars Frantoio (salt-tolerant) and Leccino (salt-sensitive). - J. Plant Physiol. 204: 8-15, 2016.

Santelia, F., Martinoia, G.: MDR-like ABC transporter AtPGP4 is involved in auxin-mediated lateral root and root hair development. - Plant Cell Physiol. 24: 5399-5406, 2006.

Shao, F., Zhang, L., Wilson, I., Qiu, D.: Transcriptomic analysis of Betula halophila in response to salt stress. - Int. J. mol. Sci. 19: 3412, 2018.

Szepesi, Á., Csiszár, J., Gémes, K., Horváth, E., Horváth, F., Simon, M.L., Tari, I.: Salicylic acid improves acclimation to salt stress by stimulating abscisic aldehyde oxidase activity and abscisic acid accumulation, and increases $\mathrm{Na}^{+}$content in leaves without toxicity symptoms in Solanum lycopersicum L. - J. Plant Physiol. 166: 914-925, 2009.

Tarkowski, Ł.P., Signorelli, S., Höfte, M.: GABA and related amino acids in plant immune responses: emerging mechanisms of action. - Plant Cell Environ. 43: 1103-1116, 2020.

Terasaka, K., Blakeslee, J., Titapiwatanakun, B., Peer, W., Bandyopadhyay, A., Makam, S., Lee, O.R., Richards, E., Murphy, A., Sato, F., Yazaki, K.: PGP4, an ATP binding cassette P-glycoprotein, catalyzes auxin transport in Arabidopsis thaliana roots. - Plant Cell 17: 2922-2939, 2005.

Thalmann, M., Pazmino, D., Seung, D., Horrer, D., Nigro, A., Meier, T., Kölling, K., Pfeifhofer, H.W., Zeeman, S.C., Santelia, D.: Regulation of leaf starch degradation by abscisic acid is important for osmotic stress tolerance in plants. - Plant Cell 28: 1860-1878, 2016.

Todd, J., Post-Beittenmiller, D., Jaworski, J.G.: KCS1 encodes a fatty acid elongase 3-ketoacyl-CoA synthase affecting wax biosynthesis in Arabidopsis thaliana. - Plant J. 17: 119-130, 1999.

Tran, T.V., Shu, F., Giles, H.E., Lambrides, C.J.: Salinity tolerance among a large range of bermudagrasses (Cynodon spp.) relative to other halophytic and non-halophytic perennial C4 grasses. - Environ. exp. Bot. 145: 121-129, 2018.

Wan, S., Wang, W., Zhou, T., Zhang, Y., Chen, J., Xiao, B., Yang, Y., Yu, Y.: Transcriptomic analysis reveals the molecular mechanisms of Camellia sinensis in response to salt stress. Plant Growth Regul. 84: 481-492, 2018.

Wang, M., Xu, Q., Yu, J., Yuan, M.: The putative Arabidopsis zinc transporter ZTP29 is involved in the response to salt stress. - Plant Mol. Biol. 73: 467-479, 2010.

Wang, M., Xu, Q., Yuan, M.: Zinc homeostasis is involved in unfolded protein response under salt stress. - Plant Signaling Behav. 6: 77-79, 2011.

Wang, Y., Gu, W., Meng, Y., Xie, T., Li, L., Li, J., Wei, S.: $\gamma$-Aminobutyric acid imparts partial protection from salt stress injury to maize seedlings by improving photosynthesis and upregulating osmoprotectants and antioxidants. - Sci. 
Rep. 7: 43609, 2017.

Xu, C., Sibicky, T., Huang, B.: Protein profile analysis of saltresponsive proteins in leaves and roots in two cultivars of creeping bentgrass differing in salinity tolerance. - Plant Cell Rep. 29: 595-615, 2010.

$\mathrm{Xu}$, Y., Burgess, P., Huang, B.: Transcriptional regulation of hormone-synthesis and signaling pathways by overexpressing cytokinin-synthesis contributes to improved drought tolerance in creeping bentgrass. - Physiol. Plant. 161: 235-256, 2017.

Yang, Y., Guo, Y.: Unraveling salt stress signaling in plants. - J. integr. Plant Biol. 60: 58-66, 2018.

Zhang, Q., Zhao, M., Qian, H., Lu, T., Zhang, Q., Liu, W.:
Enantioselective damage of diclofop acid mediated by oxidative stress and acetyl-CoA carboxylase in nontarget plant Arabidopsis thaliana. - Environ. Sci. Technol. 46: 84058412, 2012.

Zhao, S.Y., Zeng, W.H., Li, Z., Peng, Y.: Mannose regulates water balance, leaf senescence, and genes related to stress tolerance in white clover under osmotic stress. - Biol. Plant. 64: 406-416, 2020.

Zhou, M., Li, D., Li, Z., Hu, Q., Luo, H.: Constitutive expression of a miR319 gene alters plant development and enhances salt and drought tolerance in transgenic creeping bentgrass. - Plant Physiol. 161: 1375-1391, 2013. 